\title{
Study on operation method and analysis in distribution system under large-scale introduction of photovoltaic and gas cogeneration systems
}

\author{
Masanori Tokumitsu*, Shohei Haga, Yaser Qudaih, Masayuki Watanabe, \\ Yasunori Mitani
}

Department of Electrical and Electronics Engineering, Kyushu Institute of Technology, Kitakyushu-shi, Fukuoka, 804-8550, Japan

\begin{abstract}
This study assumes a case installing photovoltaics (PV) and gas cogenerations (GC) with connections to distribution system in most of houses, which makes an issue of rising voltage. Generally, rising voltage is controlled by lowering the feeder voltage by Load Ratio control Transformer (LRT). However, it cannot sufficiently operate against too many PVs in the distribution lines. In this paper analysis of the voltage profiles in this difficult situation and basic examination of operation method to solve the problem are presented. First, the simulation model representing the distribution systems in detail is introduced. Proper tap position settings to consider the sudden changes of PV generation are considered to avoid the violation of voltage limit. In addition GC power generation which can operate to support the shortage of power supply is considered to achieve a stable power system operation without voltage violation. The results, voltage deviation has occurred in the introduction rate of $80 \%$. However, the stoppage of the operation of GC at a terminal, voltage within the allowable limits is obtained.
\end{abstract}

Keywords: Photovoltaics, gas cogeneration, demand response, voltage rise

\section{Introduction}

These days, installations of renewable energy such as PV are accelerated drastically as a countermeasure for securing power supply resources and overcoming environment problems [1]. At the same time, introduction of gas cogeneration (GC) systems utilizing gas engines and fuel cells is also attracting a great deal of attentions to decrease the $\mathrm{CO}_{2}$ emissions in great amount [2], [3]. Currently, the installations of PV and GC systems in general households are spreading and they are expected to be common in near future. Here, it should be noted that distributed generation sources such as PV and GC can cause various side effects to the operation of the power system; voltage fluctuations, over-voltage, under-voltage, frequency fluctuations and so on. On the other hand, to support the shortage of power supply in peak demand hours, demand response (DR) which can manage the power consumption of the customers is being actively discussed [4]. Here, the generation from GC is available to support the power supply according to the DR request. Thus, CG can be expected as a peak power supply as well as a power supply for domestic use.

In this research, it is assumed that there are number of households with PV and GC in the distribution system. At times of high demand, the affordable power from GCs collected in the distribution system can be sent to the power system to support the stable operation of the power system. Then, the plenty amount of power generation from the customers may cause the voltage rise, which may cause voltage violation exceed the limit of $101 \pm 6 \mathrm{~V}$. This study presents the detail analysis of voltage profiles of distribution system with large amount of PVs and GCs, where the CGs operate with DR function. Some suggestions

\footnotetext{
* Manuscript received June 15, 2014; revised July 30, 2014.

Corresponding author: Masanori Tokumitsu; Tel.: +81-93-884-3243; E-mail address: m108308m@ mail.kyutech.jp.

doi: $10.12720 /$ sgce.3.4.380-384
} 
to avoid the problem are also presented.

\section{Structure of Distribution System Model}

Currently, distribution system of our country is generally high-voltage side $6.6 \mathrm{kV}$, low-voltage side $200 \mathrm{~V}$ or $100 \mathrm{~V}$. In this study, it was assumed $6.6 \mathrm{kV}$ on a high-voltage side and $100 \mathrm{~V}$ on a low-voltage side. The schematic diagram of distribution system model under study is shown in Fig. 1 [5]-[7]. It is a simplified model composed of branches and the total length is $3.91 \mathrm{~km}$. If a lot of branches exist, the data volume at the time of system analysis will increase, and the grasp in problem will become difficult. So, its branch is omitting in this simulation model. It explains as follows about this model. Node 1 is the secondary side of the transformer while node 2 is the leader terminal from the transformer. Loads and distributed generation sources are connected in node 3 to 10 . The model under study is for high-voltage distribution system and it does not take into account the low-voltage distribution system. The voltage of low voltage side is obtained by converting the high-voltage side. In general, a large power is consumed from the evening residential area. At this time, the tap position of the pole transformer was set up so that voltage might become equal at the starting point and end of a system. The tap changing position of the pole transformer is shown in Fig. 1.

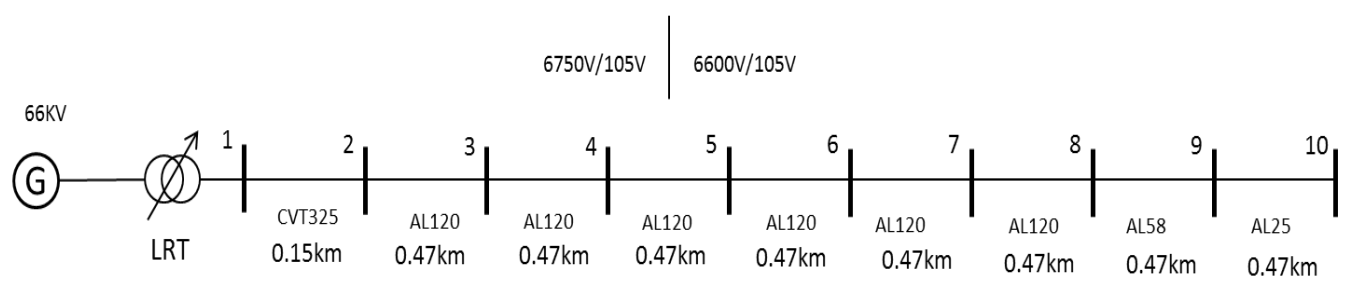

Fig. 1. Distribution system model.

\section{Method of Analysis}

In this research, the simulation of voltage rise caused by distributed generation sources is conducted with the distribution system model as shown in Fig. 1. In the distribution system model building and analysis, using MATLAB, Simulink and SimPowerSystems.

Table 1. Setting condition [10]

\begin{tabular}{cll}
\hline \hline \multirow{2}{*}{ Load } & Number of households & 800 \\
& Maximum load & $2.5 \mathrm{~kW}$ \\
& Average load (2 p.m.) & $843 \mathrm{~W}$ \\
\hline \multirow{2}{*}{ Distributed generator } & PV & $4 \mathrm{~kW}$ \\
& GC & $0.75 \mathrm{~kW}$ \\
\hline \multirow{2}{*}{ LRT } & Tapping-voltage & $60[\mathrm{~V}]$ \\
& Number of taps & 17 \\
\hline \hline
\end{tabular}

The system model is built only in high-voltage distribution system and the low voltage side is calculated from the voltage of the high voltage side as mentioned earlier. Therefore, it is necessary to consider the internal resistance of the pole transformer as well as voltage fluctuations in the low-voltage side of the distribution system. Considering a voltage variation of $3 \mathrm{~V}$ due to the resistance in the lowvoltage side, allowable voltage range becomes $101 \pm 3 \mathrm{~V}$ [8]. In addition, if the converted value from the high-voltage side is set as $101 \pm 3 \mathrm{~V}$, the voltage at the consumer terminal in the low-voltage end is also assumed to be within the acceptable range. Table 1 shows the equipment settings during simulation. LRT is a standard as for tap 0 . And, it is changeable up to \pm 8 . In other words the sending out voltage is changeable from $6120 \mathrm{~V}$ to $7080 \mathrm{~V}$. The voltage does not fall below the lower limit even when the output of PV system rapidly decreases due to sequential switching of the LRT in accordance with the 
introduction rate of distributed sources [9]. Also, it is assumed that there is no sudden change in the GC generation. Simultaneous simulation of generation from PV and GC is conducted and activation time of DR is assumed to be from 1 p.m. to 2 p.m.

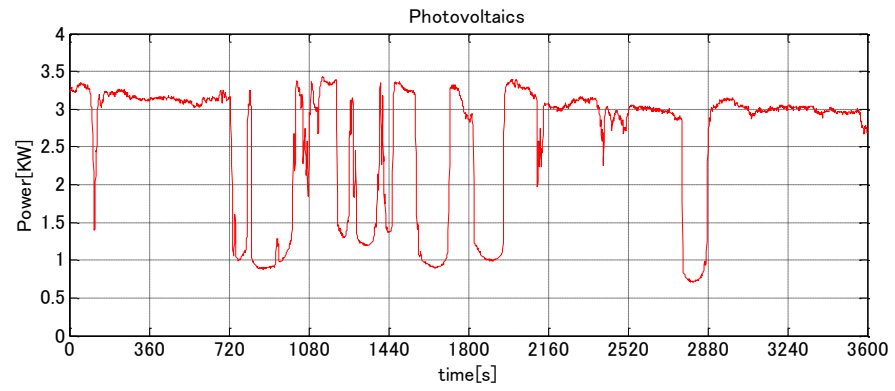

Fig. 2. PV output waveform.

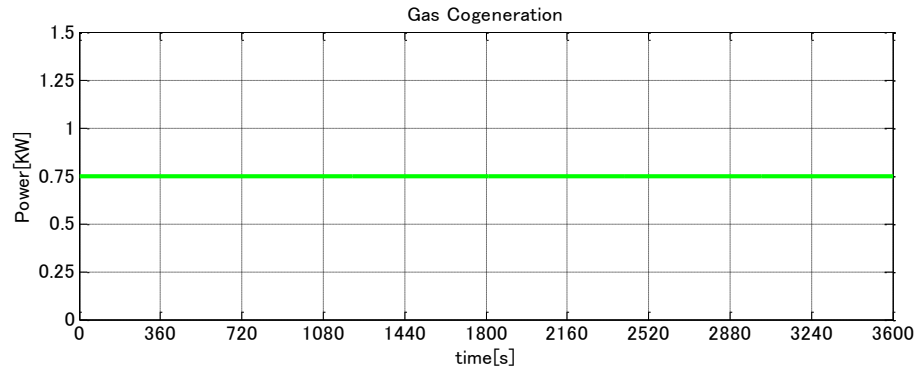

Fig. 3. GC output waveform.

\section{Analysis Result}

Currently, In the power generation capacity of the photovoltaic generation, from $3 \mathrm{~kW}$ to $3.5 \mathrm{~kW}$ is a main current. It is expected that large-capacity things spread in future. In this research, the rated output of $\mathrm{PV}$ is $4 \mathrm{~kW}$. The PV and GC output used in the analysis is shown in Fig. 2 and Fig. 3. The corresponding graph of PV is taken from the data of the $2.88 \mathrm{~kW}$ PV installed in Kyushu Institute of Technology Tobata campus and is converted to $4 \mathrm{~kW}$. The generation data of PV used the waveform from 1 p.m. to 2 p.m. on August 7, 2013. Power generation of GC used the fixed waveform of $0.75 \mathrm{~kW}$ of rate outputs.

The node voltages at 2 p.m. are shown in Fig. 4 in cases of varying introduction rate of PV and GC. This corresponds to PV and GC installed in a total of 800 households. From Fig. 4, it is seen that there is no voltage deviation in the cases of $0 \%, 20 \%$, and $40 \%$ introduction rate. This is due to the tap-changing LRT which upon anticipation of voltage rise caused by the reversed power flow, maintains the voltage not to fall below the lower limit. LRT tap position -4 is sending out voltage $6360 \mathrm{~V}$. Analysis results of the case of increasing introduction rate of addition is shown in Fig. 5. In the case of $80 \%$ introduction rate, a voltage deviation in node 10 is observed. LRT tap position -5 is sending out voltage $6300 \mathrm{~V}$. A significant voltage rise in node 9 and 10 where GC is not connected was also observed. In this case, all nodes are within the voltage limits as shown in Fig. 6.

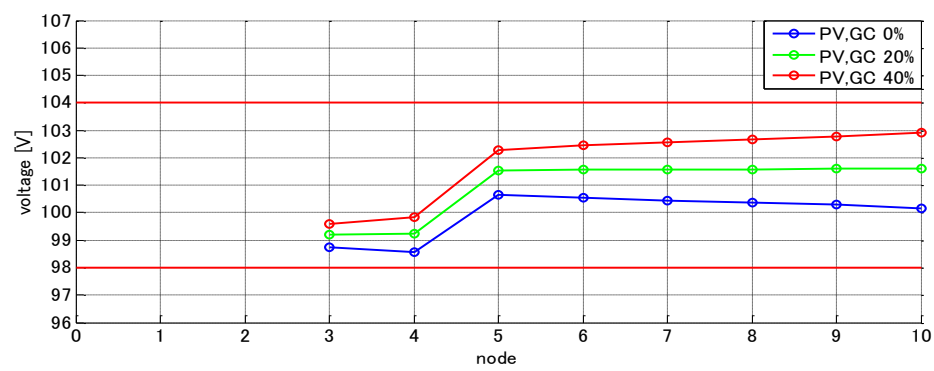

Fig. 4. LRT tap position -4 introduction rate $0 \%, 20 \%$, and $40 \%$. 


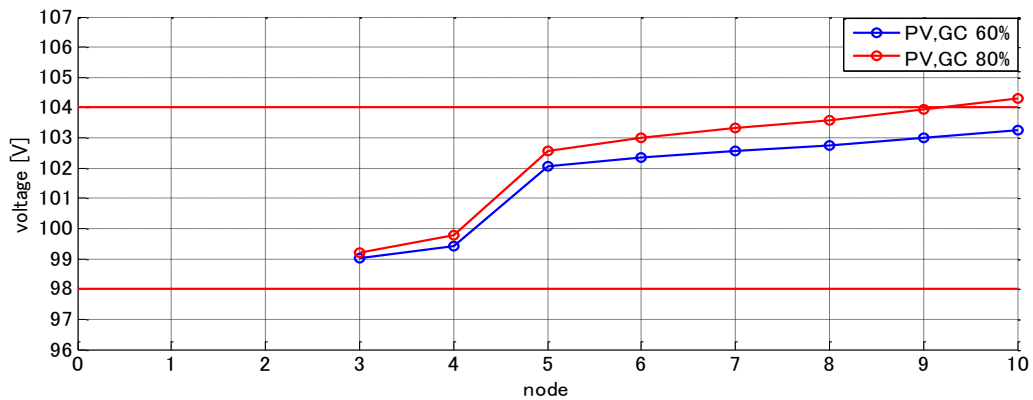

Fig. 5. LRT tap position -5 introduction rate $60 \%$ and $80 \%$.

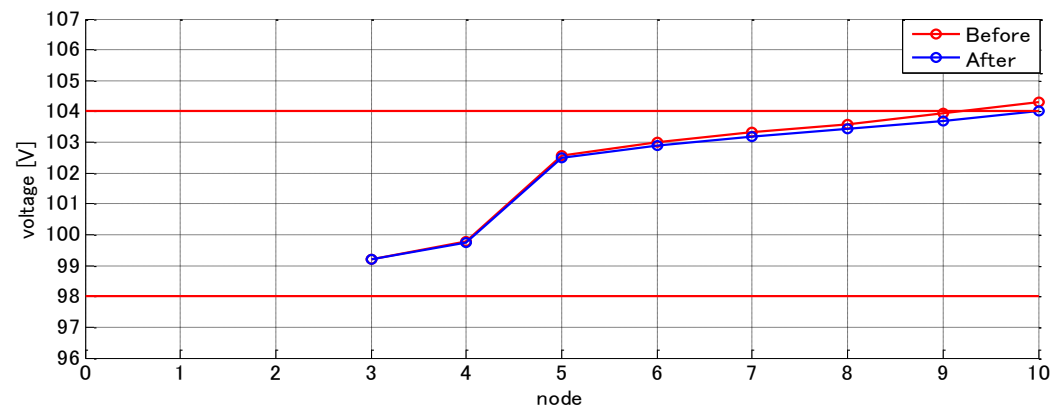

Fig. 6. Comparison before and after GC stops.

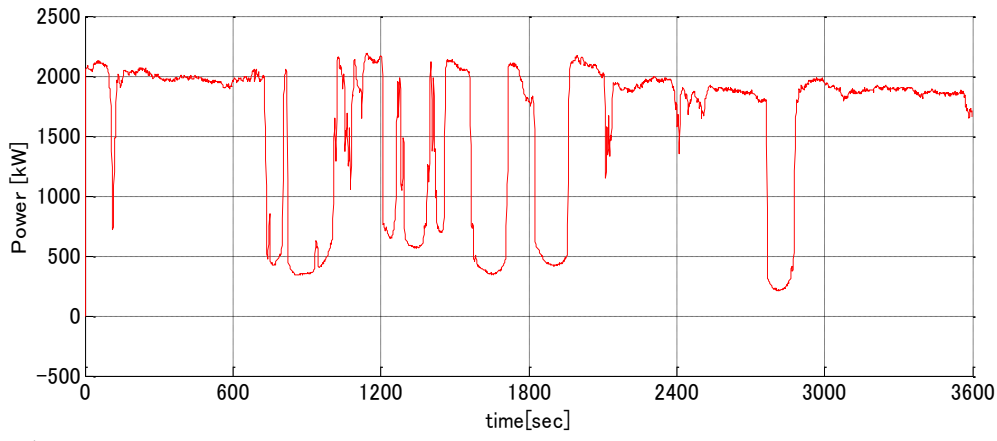

Fig. 7. Power flow to the upper system

Moreover, an analysis on the reverse power flow to the upper system subjected to DR with PV and GC generation output is conducted in the case of introduction rate of $80 \%$ and stopped generation of GC on node 9 and 10. The power flow to the upper system is shown in Fig. 7. From this result, it is known that in 1 hour time span with DR, $1691 \mathrm{kWh}$ accommodation of power to the upper system is possible.

\section{Conclusion}

In this paper, the evaluation of the large-capacity introduction of PV and GC in a distribution system in a residential area is conducted. Results obtained in this study are summarized as follows. In the system model, the sudden variations of PV are considered, and timely switching of the LRT tap-changing transformer in response to the introduction of GC and PV is observed. Voltage deviation is observed in the case of $80 \%$ introduction rate but with the stoppage of the operation of GC at a terminal, voltage within the allowable limits is obtained. However, the reverse power flow from the node with significant voltage rise is no longer possible. In addition, there can be a bias in selling power capacity depending on the distance from the substation. This disparity should be solved when the introduction of distributed generation sources advances further in the future. Moreover, the sending of power to the upper system through the reverse power flow and its contribution to the stable operation of the power system is verified. 


\section{References}

[1] Ministry of the Environment Government of Japan. Action Plan Made with Low Carbon. 2008:7-8.

[2] The Japan gas association. Promotion of Distributed Power Energy System and Shift to Natural Gas in the Energy Policy of the Future. 2012:3.

[3] Imaizumi D. Power Supply is Best Understood, Gijutsu-Hyohron; 2012:136.

[4] Kitakyushu-city environmental future city promotion office. Kitakyushu Smart Community Creation Project, 2013:25-30.

[5] Harmonics obstacle preventive measures of a distribution system. Electric Technology Research Association. 1981; 37(3):97104.

[6] MITA H, Technical consulting department, Math Works Japan. Simulation of Small-Scale Microgrid in Simulink. 2012.

[7] Hasegawa J, Oyama T, Mitani Y, Saitoh H, Kita H. Power System Engineering, The Institute of Electrical Engineers of Japan; 2002:128-129.

[8] Kawano T, Kawase T, Sekine Y, Toyota J, Matsuura K. Transmission and Distribution Engineering, Ohmsha; $1989: 201$.

[9] The Institute of Applied Energy. Progress Report relating to Power Network Technology Verification, 2006:66-68.

[10] Agency for Natural Resources and Energy. Energy White Paper 2011 - Challenges and Over the Energy. 2011:21-28. 